\title{
Understanding the Highly Dynamic Phenomena in Ultrasonic Melt Processing by Ultrafast Synchrotron X-ray Imaging
}

\author{
Jiawei Mi, Dmitry Eskin, Thomas Connolley, and Kamel Fezzaa
}

\begin{abstract}
In this paper, we present some highlighted findings from our recent research on real-time and in situ studies of the fundamentals of ultrasonic melt processing, including (1) ultrasonic bubble implosion, oscillation in liquid and semi-liquid (semi-solid) metals and their interactions with the growing solidifying phases; (2) enhanced acoustic metal flow and their impact on the liquid-solid metal interface. The real time experimental phenomena were interpreted with the aid of calculating the propagation of acoustic pressure in liquid metals using the Helmholtz equation and bubble wall pressure and velocity profile during bubble oscillation using the classical Gilmore model. The research provides unambiguous real-time evidence and robust theoretical interpretation in elucidating the dominant mechanisms of microstructure fragmentation and refinement in solidification under ultrasound.
\end{abstract}

Keywords

Ultrasonic melt processing $\quad \begin{array}{r}\text { fication } \\ \text { Solidification }\end{array}$

Synchrotron X-rays • Real-time and in situ studies

\section{J. Mi}

School of Engineering and Computer Science, University of Hull, Kingston upon Hull, HU6 7RX, UK

e-mail: j.mi@hull.ac.uk

\section{Eskin}

Brunel Centre for Advanced Solidification Technology, Brunel University London, Uxbridge, London, UB8 3PH, UK

T. Connolley

Diamond Light Source Ltd, Harwell Campus, Didcot, OX11 0DE, UK

K. Fezzaa

Advanced Photon Source, Argonne National Laboratory,

Argonne, IL 60439, USA

\section{Introduction}

In recent years, there has been a revived worldwide interest on research of ultrasonic processing (USP) of metallic alloys during solidification; and it has been driven primarily by the urgent needs of exploring and adopting environmental friendly and green technologies in metal and metallurgy industry [1]. Historically, many previous investigations on USP of metallic alloys have shown that refined, equiaxed grain structures can be produced, and therefore mechanical properties, i.e. yield strength and fracture toughness can be effectively improved [1, 2]. In addition, the soundness of castings can also be improved by USP due to reduction in shrinkage, hot tearing, and more uniform distribution of refined secondary phases and/or reduced micropores [3]. However, USP of metallic alloys has, so far, not found wide applications in industry because the exact mechanisms of how ultrasonic bubbles and acoustic flow interact with the solidifying phases in solidification, especially the highly dynamic phenomena occurring at the liquid-solid (L-S) interface have not been fully understood. This is mainly due to the opaqueness of liquid metals, which prevents direct real-time observation of the dynamic interactions between ultrasonic waves, bubbles, acoustic flow in liquid, and in semi-liquid (or semi-solid) metals. Previous studies have used water or water-based solutions [4] as well as organic transparent alloys [5] to mimic liquid metal alloys in order to study the effects of ultrasonic bubbles and streaming flow during solidification under visible light illumination. Growing dendrites were found to be fractured by oscillating bubbles or shock waves generated at bubble implosion [5]. These investigations provide valuable visual data for understanding the effects of USP. Generally, organic transparent alloys and solutions are very different from liquid metal alloys in terms of density, viscosity, surface tension, heat/mass transfer coefficient and sound velocity; and it is inappropriate to simply apply all findings from organic alloy systems to metallic systems as argued by Wang et al. [6]. 
In addition, during metal solidification, the viscosity, surface tension, sound velocity and fraction of the liquid and solid phases are governed predominantly by changes in temperature. The resulting ultrasonic bubbles and acoustic flow are also very sensitive to the thermophysical properties of different alloys and temperatures. Therefore, it is essential to study in real-time ultrasonic bubbles and acoustic flow in liquid and solidifying metals to understand fully how they interact with the liquid melts, the solidifying phases and the L-S interface. In this paper, we presented three important cases obtained from systematic in situ and real-time studies carried out in the past 8 years or so. The experiments were conducted via close collaborations with scientists of the Advanced Photon Source (APS), USA, the Diamond Light Source (DLS), UK, Synchrotron SOLEIL, France, and Swiss Light Source (SLS), Switzerland; more and systematic results have been published in [6-10]. The experimental observations and interpretations were complemented by modelling and simulation using Helmholtz equation for calculating the propagation of acoustic pressure in liquid metal; and the Gilmore model for calculating the pressure and velocity at the oscillating ultrasonic bubble wall $[6-8,10]$.

\section{Experimental Apparatus and Setup at Synchrotron X-ray Beamlines}

Figure 1 shows the experimental apparatus and the setup at the 32-ID-B of APS [6]. The sample holder was made from a quartz tube with a flat thin channel region $(15 \times 10$ $0.30 \mathrm{~mm}$ ) in the middle that can be filled with $\star 300 \mathbf{I m}$ thick liquid metal. This arrangement allows sufficient X-ray transmission through the thin layer liquid metal and to have enough space for ultrasonic bubbles to flow through freely. Feedstock alloys were melted by using a purposely-made furnace that enclosed the quartz sample holder. The dimensions of the sample holder and quartz tube, and the description of the furnace are detailed in [7]. This apparatus and the later upgraded version were used in a series of experiments carried out at a number of beamlines at APS, DLS, SOLEIL and SLS in the past six years.

\section{Bubble Implosion and Fragmentation of Dendrites}

Ultrafast imaging of ultrasonic bubble implosion experiments were conducted at the Sector 32-ID-B of APS. It is a dedicated undulator $\mathrm{X}$-ray source designed to fully utilise the 500 ns X-ray pulse by synchronizing the X-ray pulse from the storage ring with the ultrafast shutter and the camera on the beamline, delivering an ultrafast image acquisition rate of up to 271,554 fps with a spatial resolution of $1 \mathbf{l m} /$ pixel in a field of view of $1 \times 1 \mathrm{~mm}$. Synchronisation ensured that images were taken within the 500 ns exposure time, providing an image temporal resolution of $\star 500 \mathrm{~ns}$ regardless of the image acquisition rate. A Bi-8 wt\% Zn alloy was used and melted to the preset holding temperature of $427{ }^{\circ} \mathrm{C}$ (fully liquid). A Hielscher UP100H ultrasound processor $(100 \mathrm{~W}, 30 \mathrm{kHz})$ and an MS2 sonotrode (made of Ti-alloy with a tip diameter of $\varnothing 2,74 \mathrm{~mm}$ long) were used to generate ultrasonic waves inside the liquid metal. A custom-made relay trigger device was used to precisely control the application of ultrasound into the melt and the synchronisation with the high speed camera for imaging.

Figure 2 shows the evolution (changes of bubble radius with the alternating acoustic pressure) of a single bubble immediately below the sonotrode tip. The ultrasonic intensity applied was $276 \mathrm{~W} / \mathrm{mm}^{2}$ (equivalent to an ultrasound power of $20 \mathrm{~W}$ ) and the maximum acoustic pressure immediately below the sonotrode tip, i.e. $0.02 \mathrm{~mm}$ below, was calculated as $14.5 \mathrm{MPa}$ by using the Helmholtz equation [6-8]. The $\mathrm{X}$-ray images were captured using an acquisition speed of 135,780 fps. A bubble was seen in Fig. 2a-2 with a radius of $\star 8 \mathbf{~} \mathbf{l m}$ and expanded to $\star 23 \mathbf{l m}$
Fig. 1 The experimental apparatus and the setup at the 32-ID-B of APS. Detailed description are in $[6,7]$

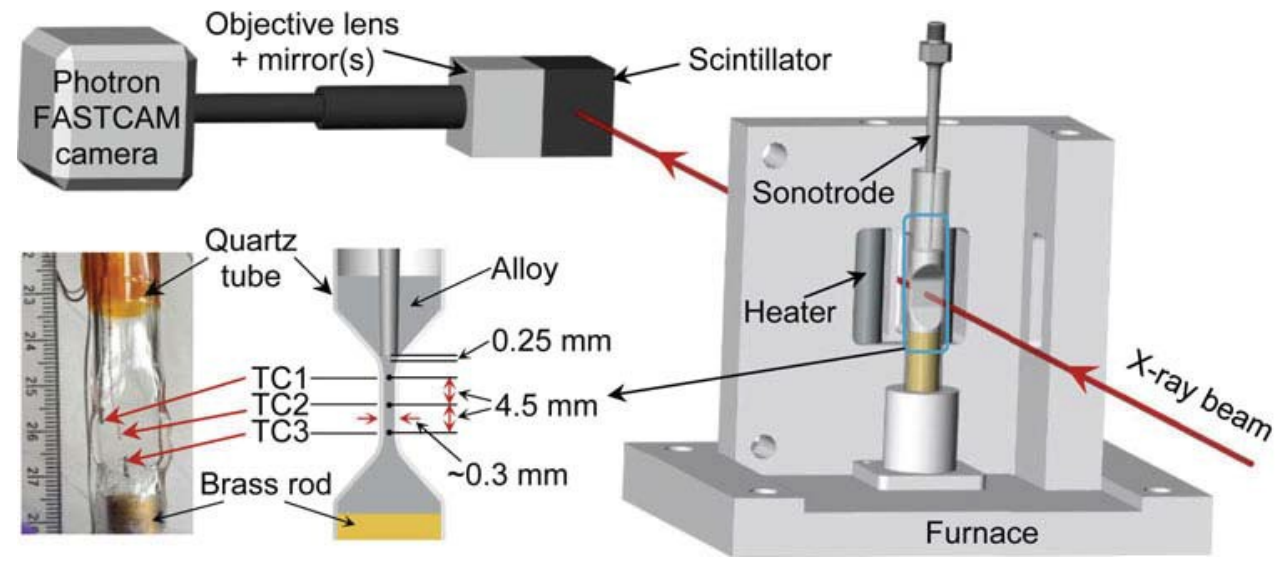


(a)
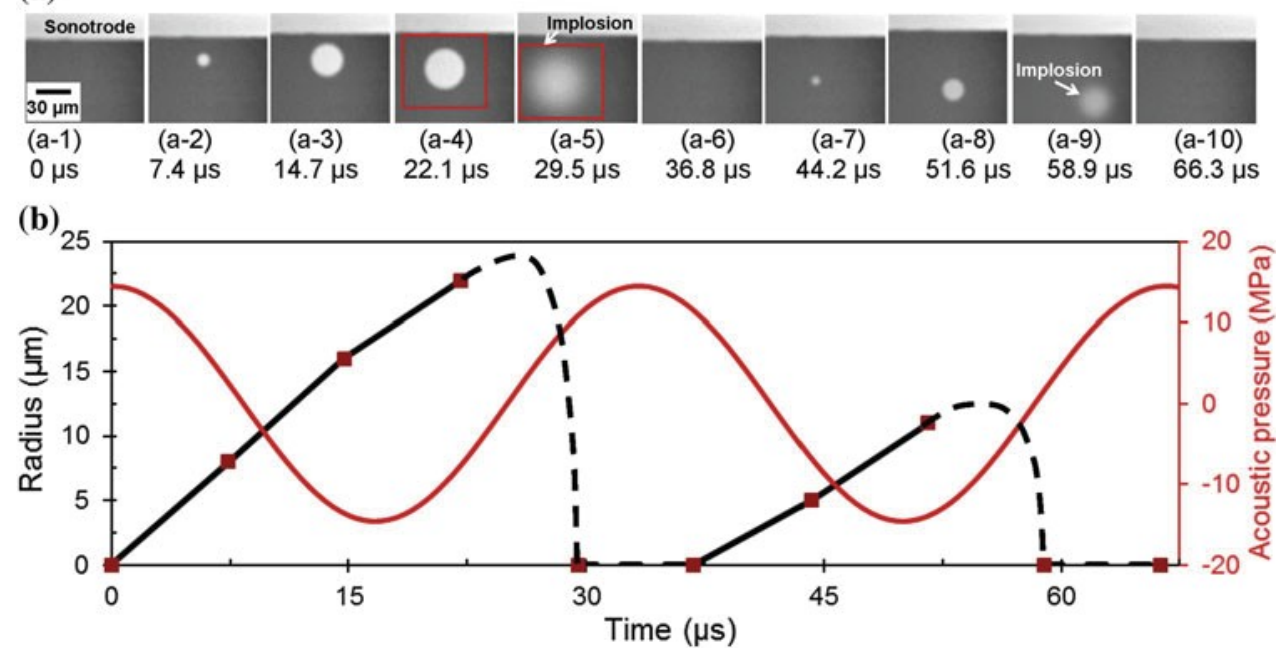

Fig. 2 a An X-ray image sequence acquired using 135,780 fps (a frame interval of $7.36 \mu \mathrm{s}$ ) at Sector 32-ID-B of APS, showing bubble implosion immediately below the sonotrode tip in a Bi-8\% Zn alloy at $427^{\circ} \mathrm{C}$; b the bubble radius measured from the images in (a), and their correlation with the acoustic pressure applied into the liquid metal. The dashed lines indicate the sudden drop of bubble radius at the instant of bubble implosion [6] in Fig. 2a-4 when the sonotrode tip moved up (the rarefaction part of the acoustic cycle). Bubble implosion occurred after the sonotrode started to move down (the compression part of the acoustic cycle), resulting in a blurred-and-misty circular region (Fig. 2a-5) with the release of high pressure shock wave to the surrounding liquid [6]. The imploded bubble produced many tiny bubbles or bubble fragments. Those with the size of $<1 \mathrm{Im}$ are too small to be seen in Fig. 2a-6 due to the spatial resolution limit of the $\mathrm{X}$-ray imaging technique. Tan [11] reported in his PhD thesis (page 126-131 of Chapter 4) many cases in water (when 271,554 fps was used) where bubble fragments of a few micrometres were observed after bubble implosion. These bubble residuals or fragments acted as nuclei for the next cycle of bubble nucleation, expansion and implosion. Many similar observations and recordings were made during the ultrafast synchrotron X-ray imaging experiments at APS. These evidences clearly indicate that, in the region below and near the sonotrode tip where acoustic pressure is sufficiently high, the whole life cycle of bubble nucleation, expansion and implosion can be completed in just one ultrasound period, i.e. 33.3 Is in this case. The bubble fragments created at bubble implosion are the overwhelming resources of the bubble nuclei for the next ultrasound period. Each implosion creates many more bubble nuclei for the next period. Such multiplication effect or chain-reaction nature are the dominant phenomenon at the region near the sonotrode tip. When the region was fully developed, an ultrasound cavitation zone was created in the liquid in which many different size bubbles oscillated very quickly and energetically, accompanied by many instances of chaotic and violent implosions of some bubbles when their diameters reach the critical threshold in the alternating acoustic pressure field. The shock wave created during bubble implosion is sufficient in shattering the nearby growing dendrites into small pieces as demonstrated by Shu et al. in [5].

\section{Bubble Oscillation and Fragmentation of Intermetallic Phases}

In situ observation of the fragmentation of primary $\mathrm{Al}_{2} \mathrm{Cu}$ intermetallic phases of an Al-35\% Cu by oscillating bubbles was conducted at the Pression Structure Imagerie par Contraste à Haute Énergie (PSICHÉ) beamline of Synchrotron SOLEIL, France [12]. The furnace was upgraded into a two-zone furnace consisting of a top and bottom halves with independent thermal control [12]. A niobium sonotrode was used due to the high melting temperature of the $\mathrm{Al}-35 \% \mathrm{Cu}$ alloy. Radiography videos were recorded by a Vision Research Miro 310 M high speed CMOS camera. A filtered pink beam with an average detected energy of $34 \mathrm{keV}$ was used. The source characteristics and imaging setup enabled a fast radiography of $1000 \mathrm{fps}$ during the application of USP to the melts, followed by slower image recording at 1 or $4 \mathrm{fps}$ during controlled cooling of the melts. The field of view was $5120 \times 2624 \mathbf{~ I m}^{2}$ at $4 \mathbf{1 m} /$ pixel. After melting the alloy, solidification process started by setting the top and bottom furnace halves to a cooling rate of $\star 2 \mathrm{~K} / \mathrm{min}$ with the temperature difference between two halves being kept unchanged. This positive thermal gradient was retained to ensure that the primary $\mathrm{Al}_{2} \mathrm{Cu}$ intermetallics grew from bottom to top. 
The sonotrode was inserted into the melt and ultrasound was applied when growing primary $\mathrm{Al}_{2} \mathrm{Cu}$ intermetallic phases were observed in the field of view. The ultrasonic transducer power was set at $100 \mathrm{~W}$ for all experiments. After $\star 5$ s of USP, the ultrasonic transducer was switched off and the sonotrode was lifted out of the melt [12].

A distinct $\mathrm{Al}_{2} \mathrm{Cu}$ phase fragmentation and detachment event observed during USP is shown in Fig. 3. The bubble cloud came into the field of view from the top left corner as shown in Fig. 3b and c. The cloud then exited the field of view at the top right corner. The passage of this bubble cloud applied a direct impact on the primary $\mathrm{Al}_{2} \mathrm{Cu}$ phases at the top left corner. One particular primary $\mathrm{Al}_{2} \mathrm{Cu}$ phase is pointed out by the dashed arrow and is circled in Fig. 3a-e. This phase was fragmented and flipped by the impact created by the passing bubble cloud. The flipped fragment displayed clear dendrite morphology with side arms as shown in the inset of Fig. 3e. Such dendritic morphology were frequently observed in the primary $\mathrm{Al}_{2} \mathrm{Cu}$ fragments during USP, and the phenomena were clearly demonstrated in the supplementary video of [12] (Video 003). After detachment, the fragment was swept out of the field of view by the acoustic streaming flow, as shown in Fig. $3 \mathrm{f}-\mathrm{i}$. Note that an individual bubble was trapped between the dendrites as marked by the solid arrow in Fig. 3f-i. It is suggested that the bubble became trapped as the main bubble cloud swept past the phases, and could not escape due to the persistent acoustic streaming.

\section{Acoustic Flow and Impact on Liquid-Solid Interface}

Real-time observation of the impact of acoustic flow on liquid-solid interface was conducted at the Beamline I12 of DLS. I12 provides synchrotron X-ray with a peak flux of $10^{12} \mathrm{ph} / \mathrm{s} / \mathrm{mm}^{2} / 0.1 \%$ bw and is a dedicated high energy instrument on a $4.2 \mathrm{~T}$ wiggler source, offering white or monochromatic X-ray beams in the range 50-150 keV. Filtered white beam was used to produce the highest possible flux without causing damage to the sample and detector. Experiments at DLS used the similar setup as showed in Fig. 1, except that the imaging system was a Vision Research Phantom ${ }^{\text {TM }} 7.3$ high speed camera, coupled to a scintillator via a $1.8 \times$ objective lens and two $90^{\circ}$ turning mirrors. Image acquisition rate used was 1500 fps with a 700 Im thick Lutetium Aluminium Garnet detector doped with Cerium (LuAG:Ce). The sample-todetector distance was $900 \mathrm{~mm}$.

A view field of $6.6 \times 5.4 \mathrm{~mm}$ with a spatial resolution of $12.2 \mathrm{Im} /$ pixel was adopted as compared to the $1 \times 1 \mathrm{~mm}$ with a spatial resolution of $1 \mathrm{Im} /$ pixel at Sector 32-ID-B of APS. The bigger field of view on Beamline I12 is therefore suitable for studying the interactions between ultrasonic bubbles/acoustic flow and the liquid-solid (L-S) interface or semisolid phases.

Systematic experiments and image sequences were taken at a deeper area below the sonotrode where acoustic flow

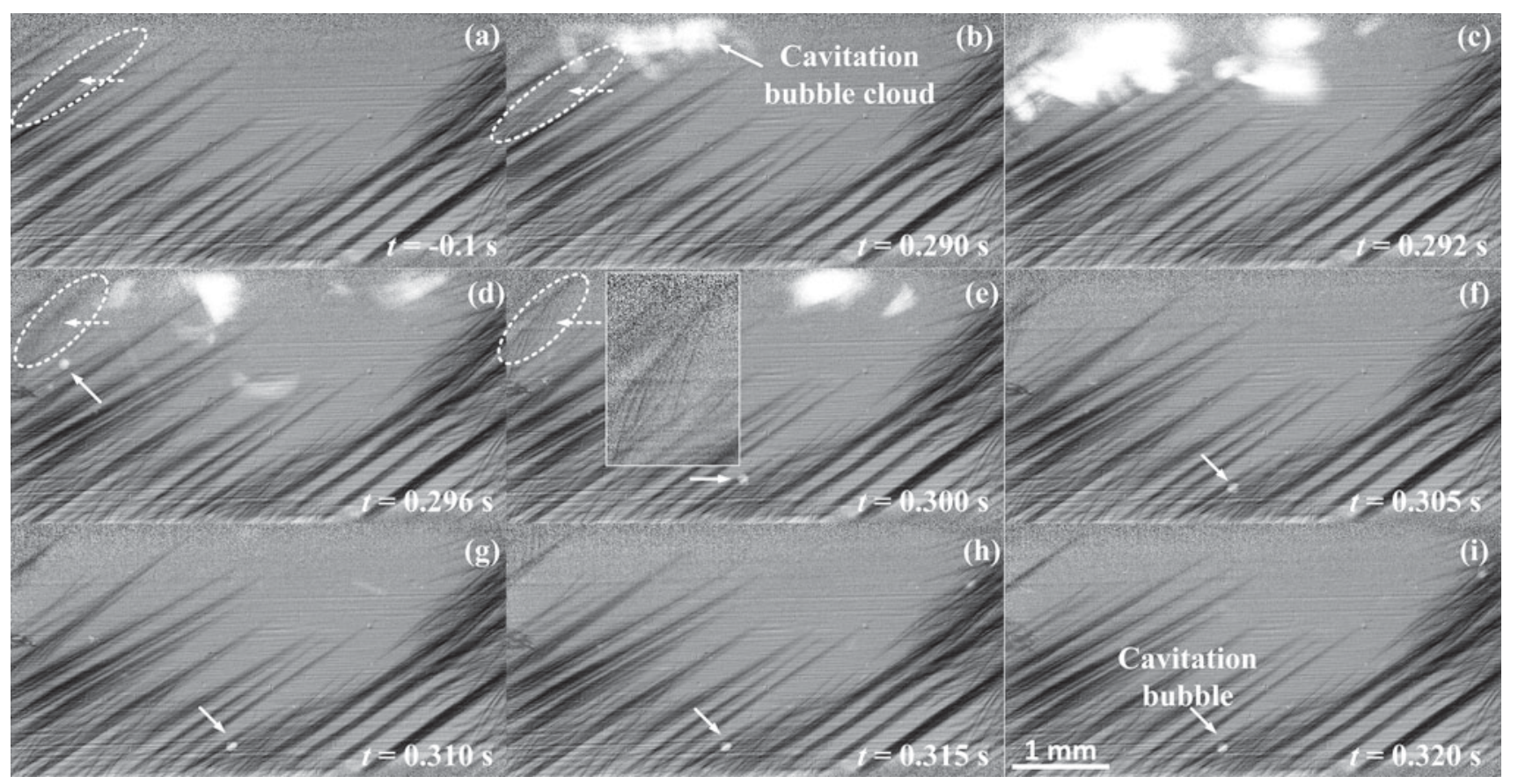

Fig. 3 Images of a passing ultrasonic bubble cloud breaking up the acoustic streaming flow. The inset in (e) is an enlarged image of the primary $\mathrm{Al}_{2} \mathrm{Cu}$ intermetallic phases at the top corner of the field of flipped dendrite indicated by the dash arrow. More details can be found view. The fragments were then swept out of the field of view by in the supplementary video in [12] 
impact on the L-S interface. Figure 4 shows the evolutions of the L-S interface under different ultrasound intensities for $2 \mathrm{~s}$ in each case. The arrows show the direction of acoustic flow (more clearly demonstrated in the video in [6]). The fluid flow pattern was found relatively symmetric to the centre of the sonotrode, clockwise on the left and anti-clockwise on the right. Figure 4a indicates that, when an ultrasound of $276 \mathrm{~W} / \mathrm{mm}^{2}$ was applied for $2 \mathrm{~s}$, a very weak acoustic flow was observed, and the L-S interface region remained almost unchanged in 2 s, with just a few 'loose' particles on the L-S interface (marked by white circle) were detached and washed away by the flow. When the intensity was increased to $926 \mathrm{~W} / \mathrm{mm}^{2}$, a strong acoustic flow was observed, causing a 'cleavage' into the solid phase at $150 \mathrm{~ms}$ (Fig. 4b). Then a big piece of the solid Zn phase was broken up and gradually washed away after $280 \mathrm{~ms}$ by the flow. The initial relatively flat L-S interface changed significantly, as indicated by the dashed lines. When $1839 \mathrm{~W} / \mathrm{mm}^{2}$ was

(a)
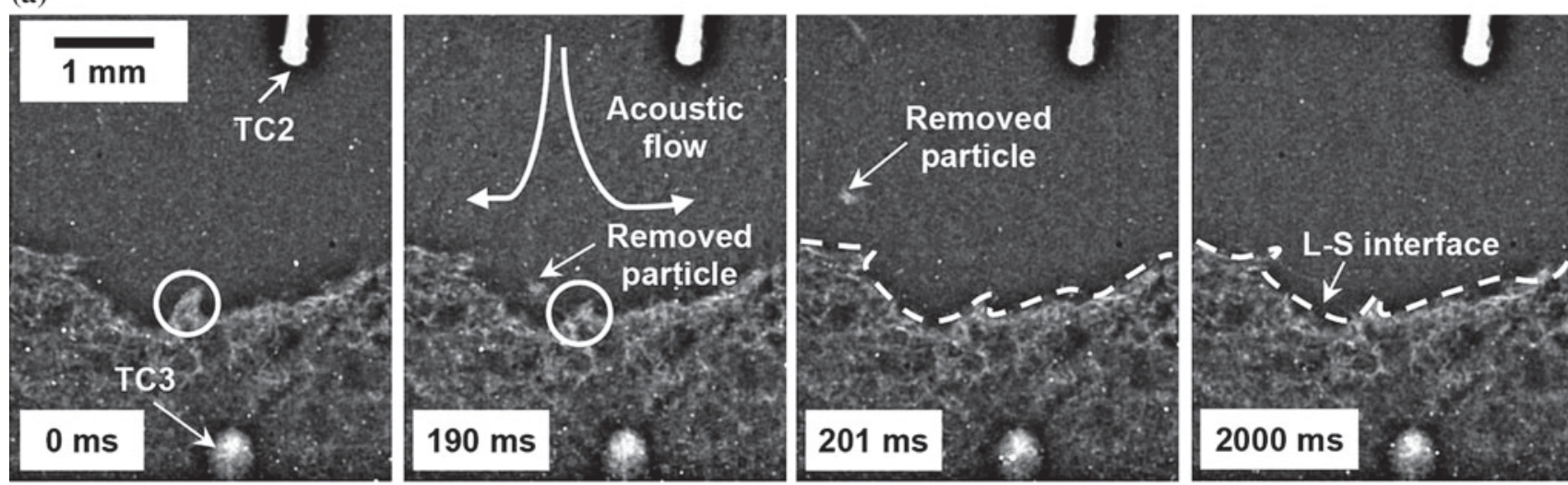

(b)
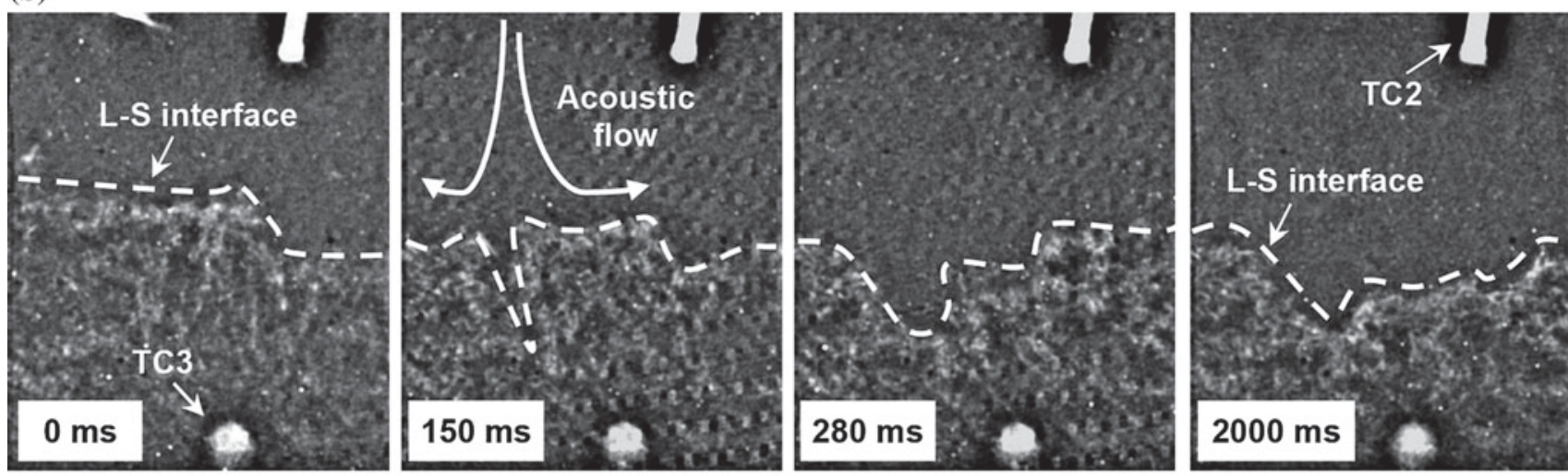

(c)
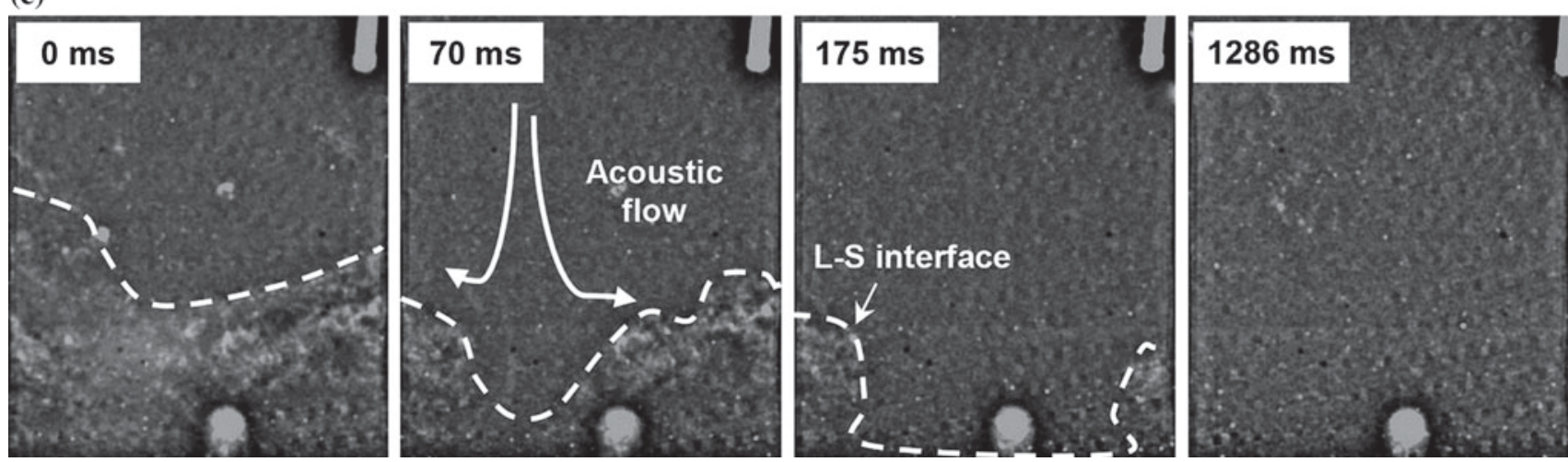

Fig. 4 Evolutions of the L-S interface of a semi-solid Bi-8\% Zn alloy under the impact of acoustic flows created by different input ultrasonic intensity: a $276 \mathrm{~W} / \mathrm{mm}^{2}$, b $926 \mathrm{~W} / \mathrm{mm}^{2}$, and c $1839 \mathrm{~W} / \mathrm{mm}^{2}$ [6].

White arrows indicate the direction of ultrasound-induced streaming flow and movement of the $\mathrm{Zn}$ phases in the liquid region; centre of images was $\star 6.5 \mathrm{~mm}$ beneath the sonotrode tip [6] 
used, Fig. 4c shows that the L-S interface was quickly broken up by the very strong swirling flow, and completely disappeared from the view field in $1286 \mathrm{~ms}$. It is also important to note that, in all cases, the detached solid particles were observed to flow back to the hotter liquid region, carried by the acoustic flow, and survived for a substantial period of time [6].

\section{Summary}

High speed and ultrafast Synchrotron X-ray imaging techniques allow the highly dynamic phenomena in ultrasound processing of metallic alloys to be studied with spatial and temporal resolution that are not possible before. We have analysed many hundreds of X-ray image video sequences acquired at carefully controlled solidification conditions with different ultrasound intensities applied. The important findings of our research are: in the well developed ultrasound cavitation zone, chaotic bubble movement and violent bubble implosion are the dominant phenomena. The imploded bubbles and the high speed acoustic flow are very efficient in breaking up the solidifying phases and the liquid-solid interface. In the region where the ultrasonic bubbles reach a quasi-steady state oscillations, the cyclic fatigue effect caused by the oscillating bubbles together with the medium acoustic flow (in the range of $0.5-0.6 \mathrm{~m} / \mathrm{s}$ [6]) are sufficient in breaking up the solidifying particles and the L-S interface.

Acknowledgements The authors would like to acknowledge the financial support from the UK Engineering and Physical Science Research Council (EPSRC) for the Ultra-Cast project [Grants Nos. EP/L019884/1; EP/L019825/1; EP/L019965/1]. The awards of the synchrotron X-ray beam time for the experiments by the Advanced Photon Source (GUP 23649 and GUP 26170), Diamond Light Source (EE8542-1 and NT12131-1) and Synchrotron SOLEIL, France (Proposal No. 20160076) are also gratefully acknowledged.

\section{References}

1. D. G. Eskin, J. Mi (eds.), Solidification Processing of Metallic Alloys Under External Fields. Springer Series in Materials Science, vol. 273. Springer, Cham, https://doi.org/10.1007/978-3319-94842-3_2.

2. G.I. Eskin, D.G. Eskin, Ultrasonic Treatment of Light Alloy Melts (second ed.), CRC Press, Boca Raton (2015).

3. X. Jian, H. Xu, T.T. Meek, Q. Han, Effect of power ultrasound on solidification of aluminum A356 alloy, Mater. Lett., 59 (2005), pp. 190-193.

4. R. Chow, R. Blindt, R. Chivers, M. Povey, A study on the primary and secondary nucleation of ice by power ultrasound, Ultrasonics, 43 (2005), pp. 227-230.

5. D. Shu, B. Sun, J. Mi, P.S. Grant, A high-speed imaging and modeling study of dendrite fragmentation caused by ultrasonic cavitation, Metall. Mater. Trans. A, 43 (2012), pp. 3755-3766.

6. B. Wang, D. Tan, T.L. Lee, J.C. Khong, J. Mi, Ultrafast synchrotron X-ray imaging studies of microstructure fragmentation in solidification under ultrasound. Acta Mater. 144, 505-515 (2018).

7. D. Tan, T.L. Lee, J.C. Khong, T. Connolley, K. Fezzaa, J. Mi, High speed synchrotron X-ray imaging studies of the ultrasound shockwave and enhanced flow during metal solidification processes. Metall. Mater. Trans. A 46, 2851-2861 (2015).

8. J. Mi, D. Tan, T.L. Lee, In situ synchrotron X-ray study of ultrasound cavitation and its effect on solidification microstructures. Metall. Mater. Trans. B 46B, 1615-1619 (2015).

9. F. Wang, D. Eskin, J. Mi, C. Wang, T. Connolley, A synchrotron $\mathrm{X}$-radiography study of the fragmentation and refinement of primary intermetallic particles in an Al-35 $\mathrm{Cu}$ alloy induced by ultrasonic melt processing. Acta Mater. 141, 142-153 (2017).

10. S. Wang, Z. Guo, J. Mi, In situ high speed imaging study and modelling of the fatigue fragmentation of dendritic structures in ultrasound fields, Acta Mater. 165, 388-397 (2019).

11. D. Tan, In situ ultrafast synchrotron X-ray imaging studies of the dynamics of ultrasonic bubbles in liquids, $\mathrm{PhD}$ Thesis, University of Hull, United Kingdom (2015).

12. F. Wang, D. Eskin, J. Mi, C. Wang, B. Koe, A. King, C. Reinhard, T. Connolley, A synchrotron X-radiography study of the fragmentation and refinement of primary intermetallic particles in an Al-35 Cu alloy induced by ultrasonic melt processing, Acta Materialia, Vol. 141 (2017), 142-153. 